and TCL genomic DNA derived from RIP-Tag mice are being used for CGHa to analyse DNA copy-number loss and to identify BAC clones consistently deleted in the $\mathrm{LOH}$ regions of interest.

Hoffman, Eric

\section{Expression profiling in human muscle disease: identification of early transcrip- tion changes consequent to dystrophin deficiency, and design of a human mus- cle tissue oligonucleotide array chip}

\section{Eric P. Hoffman'1, Yi-Wen Chen'1, Rehannah Borup ${ }^{1}$, Giorgio Valle ${ }^{2}$ \& Gerolamo Lanfranchi ${ }^{2}$ \\ ${ }^{1}$ Research Center for Genetic M edicine, Children's National M edical Center, Washington, DC 20010, USA \\ ${ }^{2}$ CRIBI Biotechnology Centre, University of Padova, Italy}

Expression array analyses promises to greatly expedite our understanding of the molecular pathophysiology of inherited muscle disease, and to assist in the identification of new disease genes through locus-specific decreases in loss-of-function (recessive) inherited dystrophies. Toward this end, we have initiated a series of expression array analyses of human muscular dystrophy patient muscle biopsies. We have chosen Affymetrix GeneChip technologies for the following reasons: (i) muscle contains large numbers of related, yet functionally diverse, genes (for example $>10$ myosin heavy chain genes), which are not distinguished by cDNA array methods; (ii) muscle is a relatively simple and homogeneous tissue which seems particularly amenable to expression array approaches and (iii) excellent non-normalized human muscle EST database has been produced by the Telethon EST project in Italy, with approximately 1,000 genes, 1,500 EST clusters and 1,500 EST singletons currently characterized.

Duchenne muscular dystrophy is the most common inherited lethal childhood disease in the world, and is equally frequent in animals. The gene is approximately 2.5 megabases, and has a new mutation rate of 1 in 10,000 gametes. Dystrophin deficiency is the biochemical cause, and this deficiency is expressed from fetal life (16 weeks gestation) onward, yet clinical symptoms are not obvious until 4-5 years of age. The reasons underlying the progressive pathophysiology are not understood. Here, we describe initial experiments using human fetal muscle samples from a normal fetus, and a DMD fetus, using Affymetrix stock human 6,800 chips. DMD comparison of signals from fluorescent cRNA samples was done using Affymetrix data analysis for significant differences. Ninety-nine percent $(7,062 / 7,129)$ of the probe sets tested showed similar levels of cRNA between the control and DMD samples. Seventeen $(0.24 \%)$ of the probe sets showed significant decreases in expression. These decreases included an important dystrophinassociated protein, beta-dystroglycan, and proteins involved in immune recognition of abnormal cells (tenascin $\mathrm{X}$ and macrophage migration inhibitory factor $[\mathrm{MIF}])$. Our data show that secondary transcriptional changes occur soon after dystrophin deficiency is biochemically evident, long before clinical symptoms appear. This data will permit the delineation of the molecular pathophysiological progression of this important disorder, and provide insight into the development of the membrane cytoskeleton of muscle.

Our long-term goals are to construct an extensive database of expression array changes in muscular dystrophy patients, with known variables of patient age at time of muscle biopsy, and molecular diagnosis (gene and gene mutation). By constructing a gene- and age-specific expression array database, we anticipate that novel muscular dystrophy genes can be identified through specific changes in single RNAs in unknown samples. Toward this end, we have under production an Affymetrix human muscle chip, which uses the non-normalized muscle EST database of the University of Padova, Italy. The chip will have 3,000 genes/clusters/singletons ranked from most to least abundant on the chip, with the usual 40fold redundancy (120,000 features). The informatics should ensure the ability to distinguish between closely homologous muscle genes, which would be impossible using cDNA array technology.

Hoheisel, Jörg

\section{Standardisation of DNA-array analyses}

\author{
Jörg D. Hoheisel
}

Deutsches Krebsforschungszentrum, Im Neuenheimer Feld 506, D-69120 Heidelberg, Germany

Imperative for a sensible use of DNA-chips is the establishment of standardised procedures and processes for a comparable and compatible data production, quality control and analysis. To this end, we have established a surface chemistry for the covalent attachment by both immobilisation and in situ synthesis of DNA molecules on glass or polypropylene. Apart from stable bonding of more than 30 consecutive hybridisations, parameters like loading capacity, charge and hydrophobicity can be modified in a controlled manner. For photolithographic oligomer synthesis, we developed a chemistry that yields quantitative efficacy of each condensation reaction. In addition, a procedure was set up allowing for an accurate measurement of yield and amount at all individual positions of every oligomer chip prior to subsequent uses of the very chip. Substitution of PNA for DNA molecules on the arrays combined the high selectivity of oligomers with the strong duplex stability of PCR-products at hybridisation conditions which prevent double-strand formation between the DNA probes. Lastly, we are working on technology to replace optical detection by a system not requiring labelling nor PCR-amplification of probe material even from limited samples prior to quantitative on-line detection.

Holden, Peter

\section{Toxicogenomics: the application of microarrays to toxicology}

\author{
P.R. Holden \& W.D. Pennie \\ AstraZeneca Central Toxicology Laboratory, Alderley Park, \\ Cheshire, SK10 4TJ, UK
}

Microarray technology provides an exciting new approach to testing and predicting the toxicity of compounds as well as understanding their mode of action. We have constructed a number of custom microarrays specifically designed to investigate the transcriptional activation of genes by toxic compounds. Around 600 cDNA clones from human and mouse libraries were chosen because of their relevance to toxicology. They were PCR-amplified, purified and immobilised onto nylon membranes to generate species-specific toxicogenomics arrays. A detailed database on biochemical function, role in disease and allelic variation has been assembled for each gene on the array to facilitate interpretation of the data. These arrays have been used to investigate the mechanistic aspects of a number of toxic endpoints such as hepatotoxicity, endocrine disruption and carcinogenesis. We are also using these arrays to determine the toxicity of compounds on the basis of their gene expression patterns. The results of these experiments will be presented and the broad application of genomics to toxicology will be discussed. 